\title{
ANÁLISIS DE FACTORES CONTEXTUALES AMBIENTALES EN RELACIÓN AL DESEMPEÑO OCUPACIONAL. LA INCLUSIÓN DESDE LA PERSPECTIVA DE PERSONAS EN SITUACIÓN DE DISCAPACIDAD EN LA CIUDAD DE MAR DEL PLATA, ARGENTINA DURANTE EL PERÍODO 2017-2018
}

\author{
ANALYSIS OF ENVIRONMENTAL CONTEXTUAL FACTORS IN RELATION TO \\ OCCUPATIONAL PERFORMANCE. INCLUSION FROM THE PERSPECTIVE OF PEOPLE \\ WITH DISABILITIES, IN MAR DEL PLATA, ARGENTINA, DURING 2017-2018
}

\section{María Alejandra Campisi', Verónica Anahí Fernández ${ }^{2}$}

\begin{abstract}
RESUMEN
La presente investigación tuvo como objetivo analizar los factores contextuales ambientales que se vinculan al desempeño ocupacional de personas en situación de discapacidad en las áreas trabajo / estudio y tiempo libre / ocio (actividades recreativas); y a partir de estos resultados comprender sus vivencias de inclusión social. Se propuso un diseño transversal exploratorio y descriptivo, empleando una metodología cualitativa que permitiera analizar, comprensiva e interpretativamente, la perspectiva del sujeto estudiado a partir de escenarios particulares. El grupo de estudio, seleccionado por conveniencia, se conformó de veinte personas en situación de discapacidad, cuyas edades oscilaron entre 19 y 6 o años. Los datos se obtuvieron a través de entrevistas estandarizadas no programadas. Los resultados más destacados indican que existen factores contextuales ambientales, físicos y sociales que interfieren en el desempeño ocupacional autónomo de las personas del grupo estudiado; especialmente barreras arquitectónicas, ausencia de dispositivos tecnológicos de ayuda, falta de cumplimiento de legislación vigente y en algunos casos las actitudes negativas de las personas del entorno inmediato, ya sea educativo como comunitario. De las experiencias subjetivas relatadas emerge el sentido de inclusión como la capacidad de sentirse productivo y autónomo en igualdad de condiciones. Quedan abiertas varias líneas para continuar investigando, vinculadas a cada uno de los factores estudiados y a la concepción de inclusión.
\end{abstract}

\section{PALABRAS CLAVE}

Personas con discapacidad, educación, trabajo, actividades recreativas, Terapia Ocupacional

1 Licenciada en Terapia Ocupacional. Especialista en Bioética. Especialista en Docencia Universitaria. Universidad Nacional de Mar del Plata. Docente regular-Investigadora categorizada. Facultad de Ciencias de la Salud y Trabajo Social, Universidad Nacional de Mar del Plata. Vicedirectora de la Escuela de Educación Escuela Asociación Manuel Belgrano DIEGEP 661 - El Portal del Sol, Mar del Plata, Provincia de Buenos Aires, República Argentina. Correo electrónico: mariaalejandracampisi@gmail.com Correo postal: Florencio Sánchez 265, (7600) Mar del Pata, Provincia de Buenos Aires, República Argentina. Teléfono: 542236824194

2 Licenciada en Terapia Ocupacional. Especialista en Docencia Universitaria. Universidad Nacional de Mar del Plata. Docente regularInvestigadora categorizada. Facultad de Ciencias de la Salud y Trabajo Social, Universidad Nacional de Mar del Plata. Correo electrónico: vafernan@mdp.edu.ar

Correo postal: Chacabuco 5189, (7600) Mar del Plata, Provincia de Buenos Aires, Argentina. Teléfono: 542234740873 


\begin{abstract}
The objective of this research was to analyze the environmental contextual factors that are linked to the occupational performance of people with disabilities in the areas of work/study and leisure time/leisure; and from these results to understand their experiences of social inclusion. An exploratory and descriptive cross-sectional design was proposed, using a qualitative methodology that allowed analyzing, comprehensively and interpretively, the perspective of the subject studied from particular scenarios. The study group, selected for convenience, was made up of twenty people with disabilities, whose ages ranged between 19 and 6 o years. The data was obtained through standardized unscheduled interviews. The most outstanding results indicate that there are environmental, physical and social contextual factors that interfere in the autonomous occupational performance of the people of the group studied; especially architectural barriers, absence of technological aid devices, lack of compliance with current legislation, and in some cases, the negative attitudes of people in the immediate environment, whether educational or community. From the subjective experiences reported, the sense of inclusion emerges as the ability to feel productive and autonomous under equal conditions. Several lines are open to continue research, linked to each of the factors studied and the conception of inclusion.
\end{abstract}

\title{
KEYWORDS
}

Disabled Persons, education, work, leisure activities, occupational therapy

Recibido: 18 Marzo 2019

Aceptado: 02 Diciembre 2019

\section{INTRODUCCIÓN}

Este estudio se enmarca en el trabajo que realiza el grupo de investigación "Discapacidad, ocupación y contexto social" de la Universidad Nacional de Mar del Plata (Provincia de Buenos Aires, República Argentina) que, en los últimos años, ha investigado temáticas vinculadas a los procesos de inclusión de las personas en situación de discapacidad, analizándolos en las áreas ocupacionales de tiempo libre / ocio, educación, productividad. Continuando esta línea de investigación, se elabora un nuevo marco conceptual y problemático basado en la necesidad de profundizar en el proceso de inclusión, abordando otros aspectos que hacen al mismo y que aún no habían sido analizados, enfocándose en los factores contextuales ambientales que facilitan u obstaculizan la inclusión de personas en situación de discapacidad y, por consiguiente, el desempeño ocupacional en su sentido más amplio.

Desde el Modelo Social de Discapacidad, se conceptualiza a la discapacidad como un fenómeno multidimensional resultante de un proceso dialéctico, generado en la interacción dinámica de la persona con su entorno. La discapacidad es comprendida, entonces, como una construcción social y como un aspecto más de la diversidad que caracteriza a la especie humana (Palacios, 2008). Esta perspectiva implica pensar en una sociedad accesible para todos, que incluya las modificaciones ambientales, actitudinales y culturales necesarias para el logro de la inclusión y la acción política que posibilite igualdad de derechos y oportunidades para las personas con discapacidad en todas las áreas de la vida social.

Un avance significativo en cuanto a la discapacidad como una cuestión de derechos humanos, surge con la Convención Internacional sobre los Derechos de las Personas con Discapacidad promulgada por las Naciones Unidas (ONU, 2006) y ratificada por la República Argentina en el año 2008 (Ley Nacional 26.378), que impulsa la promulgación de normas administrativas y jurídicas por parte de los Estados y la puesta en marcha de proyectos, planes y programas que ofrezcan garantías de participación, calidad de vida y plena inclusión social.

La Clasificación Internacional del Funcionamiento, de la Discapacidad y de la Salud -CIF- (oms, 2001) hace referencia a un nuevo Modelo Biopsicosocial de la Discapacidad, entendiendo que la misma no es la característica de algún grupo social sino que se trata de una experiencia humana universal, un concepto dinámico resultante de la interacción entre el estado de salud personal y los factores contextuales.

Se describen por primera vez en esta "clasificación", los Factores Contextuales Ambientales, que impactan en la salud y constituyen el trasfondo total de la vida de una persona. Los factores ambientales constituyen el ambiente físico y social de la vida cotidiana; son externos a la persona y pueden intervenir de modo positivo o negativo en su desempeño y participación en la comunidad. Los factores ambientales a nivel individual hacen 
referencia al entorno inmediato de la persona, sean los espacios físicos como las relaciones personales que en ellos establece; en tanto a nivel social, son las estructuras sociales formales e informales, los servicios y sistemas existentes en una sociedad o la cultura en la misma, tales como las organizaciones, normas, actitudes, etc.

Un aporte a esta perspectiva son los conceptos del Modelo de Ocupación Humana de Kielhofner (2004), que concibe al ser humano como un sistema abierto en constante interacción con el ambiente, en una relación dialéctica, dinámica y compleja en donde ambos se modifican y construyen uno al otro, transforman y se transforman. Desde este modelo, los contextos ambientales son una combinación de espacios naturales y artificiales, los objetos que en ellos disponemos, las formas ocupacionales, los grupos sociales y la cultura. Lo físico y lo social se hallan entrelazados en cada ambiente que encontramos y juntos constituyen los contextos del comportamiento ocupacional, los cuales representan una jerarquía ambiental que influye en la selección de nuestros espacios de relación y en los desempeños ocupacionales subsecuentes.

Los factores contextuales tienen efecto en la condición de salud, constituyendo el escenario de interacción donde las posibilidades de inclusión de las personas con discapacidad pueden verse favorecidas o restringidas (OMS, 2001).

El desempeño ocupacional depende de los factores contextuales, siendo esencial para el logro de autonomía, autorrealización y participación, incidiendo además en las oportunidades de inclusión social.

La inclusión social constituye un concepto complejo, que abarca aspectos culturales, históricos, contextuales. No es un fin en sí misma, sino un medio a través del cual potenciar al máximo la participación. La inclusión supone la transformación de las barreras estructurales, que impiden el cambio, en oportunidades de participación social. Dichas barreras físicas y culturales abarcan las concepciones predominantes de éxito y de fracaso y se ponen de manifiesto en las prácticas sociales, sean educativas, institucionales asistenciales, laborales, etc. Es por ello que este proceso de inclusión es crítico, desafiante y perturbador, al intentar comprender la diferencia en forma constructiva. Tal como expresa Ainscow (citado por Barton, 2009): "es un proceso público de designación y celebración de las diferencias así como de identificación con aquello que apreciamos los unos de los otros. Construir un ámbito de igualdad, en el que las diferencias sean una oportunidad para aprender, en lugar de ser un problema a excluir" (p. 133).

Existe, entonces, una relación dinámica entre estos elementos, aspecto posible de indagación, siendo relevante recoger datos de estos constructos a fin de profundizar y ampliar el conocimiento existente sobre el proceso de inclusión y los aspectos que impactan positivamente en el mismo.

Por tanto, entendiendo que el desempeño ocupacional en las áreas de autocuidado, trabajo, estudio y esparcimiento es esencial para la inclusión social y que estas cuestiones dependen de los factores ambientales en los que interactúa la persona con discapacidad, el objetivo del presente estudio es analizar los factores contextuales ambientales que se vinculan al desempeño ocupacional de personas en situación de discapacidad en el Partido de General Pueyrredon en el período 2017-2018; a partir de estos resultados comprender sus vivencias de inclusión social.

\section{MÉTOdo}

Teniendo en cuenta la naturaleza de los objetivos, se propone un estudio exploratorio-descriptivo con un diseño transversal, empleando metodología cualitativa por entender que mediante la misma, la investigación se transforma en un proceso progresivo de construcción y creación, donde los distintos momentos son analizados comprensiva e interpretativamente, en primera instancia desde la perspectiva del sujeto estudiado a partir de escenarios particulares, pudiendo descubrir a posteriori principios más generales al considerar el análisis del contexto físico, social y cultural en el cual ocurre la interacción entre factores ambientales, desempeño y participación.

El grupo de estudio se conformó de veinte personas en situación de discapacidad, cuyas edades oscilaron entre 19 y 60 años, heterogéneo en cuanto a sexo, tipo de discapacidad (física, mental, intelectual y sensorial) y tipo de apoyos requeridos (silla de ruedas, audífonos, andador, bastones diversos). Cinco de los participantes del estudio concurrían a escuelas de educación especial y realizaban cursos de formación profesional o experiencias de pasantías laborales. La mayoría había 
culminado los estudios primario o secundario y algunos se encontraban cursando estudios terciarios y universitarios (ver Tabla 1).

TABLA 1

NIVEL DE ESTUDIOS DE LOS INTEGRANTES DEL GRUPO DE ESTUDIO SELECCIONADO, PERÍODO 2017-2018

\begin{tabular}{|l|l|}
\hline Nivel de estudios & $\mathrm{N}^{\mathrm{o}}$ \\
\hline Primario & 6 \\
\hline Secundario & 6 \\
\hline Educación Especial & 5 \\
\hline Terciario/Universitario & 3 \\
\hline Total participantes & 20 \\
\hline
\end{tabular}

Autoría propia

Cada participante consintió en forma verbal su participación en el estudio, preservando su anonimato y privacidad a fin de respetar los estándares de la ética de la investigación a lo largo de todo el proceso.

Los datos se obtuvieron a través de entrevistas estandarizadas no programadas dado que se trabajó con una guía de preguntas formuladas y secuenciadas de modo tal, en la situación concreta de cada entrevista permitieran el flujo de información. Se desarrollaron a modo de diálogo y se generó una conversación acerca de cuestiones que resultan cotidianas para el/la entrevistado/a (Valles, 1999).

Los ejes de las entrevistas fueron los factores contextuales personales, ambientales físicos y sociales y sus vinculaciones con el desempeño ocupacional. A medida que se analizaban los datos, se incorporó como categoría de análisis el concepto de inclusión desde la perspectiva de las propias personas en situación de discapacidad, sus experiencias y vivencias cotidianas.

Los y las participantes entrevistados fueron seleccionados entre aquellas personas en situación de discapacidad, que eran accesibles para los investigadores por conocimiento y contacto personal de los integrantes del grupo de investigación de la Facultad de Ciencias de la Salud y Trabajo Social. Las entrevistas se Ilevaron a cabo en los entornos cotidianos o ambientes de pertenencia de las personas en situación de discapacidad, Centros de día, ONG, instituciones educativas, asistenciales, ámbito laboral, entre otros, relacionados con la discapacidad.

Para el tratamiento de los datos se realizó análisis de contenido (Coffey, 2003) por ser un método que posibilita el análisis del discurso narrativo, a fin de comprender e interpretar los testimonios de los participantes. Se entiende que este método permite captar, sintetizar, interpretary re-contextualizar, formulando inferencias a partir de los datos, en un proceso continuo, significante y creativo.

\section{Resultados}

El funcionamiento de las personas se interpreta por la interacción entre los estados de salud y los factores contextuales (personales, ambientales) que actúan como facilitadores o como barrera en el ámbito físico, social, actitudinal (OMS, 2001).

Los apoyos apropiados y oportunos permiten la independencia de la persona. Se entiende por independencia al control que cada uno tiene sobre su propia vida y a la calidad de vida lograda, más allá de la necesidad de asistencia requerida (Palacios, 2008).

En este marco se realiza un análisis que aproxime al conocimiento de los factores contextuales en el grupo de estudio que favorecen la inclusión y el desempeño ocupacional.

\section{Los factores contextuales personales}

Los factores personales se refieren a las características del individuo como sexo, edad, estados de salud, estilos de vida, hábitos, nivel de educación, experiencias vitales, intereses, personalidad, aspectos psicológicos, profesión, entre otras, que no forman parte de una condición o estado de salud; si bien pueden desempeñar un rol en la discapacidad, no están clasificados en la CIF debido a la gran variabilidad cultural y social que presentan.

En este estudio, no obstante, se pudieron recabar datos acerca los aspectos mentales, psicosociales, del temperamento y la personalidad desde la propia perspectiva de las personas que formaron parte del grupo de 
estudio, quienes pudieron describir cómo se perciben a sí mismas, relacionando sus propias características personales con la sensación de sentirse incluidos o no en su entorno social:

"Cuando la persona logra autonomía, es positi$v a$, sociable, agradable, tiene mayores posibilidades de insertarse. Cuando es inquieta y busca las oportunidades y además tiene el apoyo de sus seres queridos, tiene más oportunidades." (E2, mujer -m-, 21 años)

"El estado de ánimo estable y constante como es un factor importante." (E1, m, 43a)

El estado de ánimo positivo, el involucramiento en las situaciones sociales, el sentido de compromiso y responsabilidad, el desarrollo de autonomía, son algunos de los factores manifestados con mayor frecuencia que favorecen la inclusión. Estos se presentan en un entramado familiar, comunitario y social, en el que se interrelacionan y se potencian o no.

\section{Los factores contextuales ambientales físicos. El problema de la accesibilidad.}

Las personas con movilidad reducida se enfrentan a obstáculos en espacios públicos para actividades de esparcimiento, laborales, educativas. La presencia de barreras urbanas o arquitectónicas y la ausencia de apoyos tecnológicos suponen la necesidad de acompañantes y cuidadores. En otros casos, la posibilidad de independencia y participación depende de la solidaridad de terceros:

"Casi siempre lo que me ayuda a salvar los obstáculos es la persona que me acompaña o tres o cuatro que andan por ahi, que se solidarizan y me dan una mano." (E8, hombre -h-, 40 años)

"El ambiente tiene barreras físicas ya que no está preparado para incluir a la persona con discapacidad. Los lugares en general no cuentan con elementos de ayuda. (...) Ir a cualquier lugar público... siempre voy acompañada."(E9, m, 22a)
Los diseños arquitectónicos en los que se movilizan las personas del grupo de estudio, en general, presentan barreras. El ambiente físico no cuenta con ayudas tecnológicas (traductores para el caso de personas con hipoacusia), esto sumado a algunas actitudes sociales dificulta cualquier intento de desempeño independiente y de calidad de participación social e incluso la impiden:

"El ambiente físico no está preparado, no hay rampas, no hay alarmas sonoras, se carece de elementos que favorezcan la inclusión (...) El ambiente físico limita las actividades, ya que no podes desenvolverte en forma totalmente autónoma."(E10, m, 35a)

"Faltan en la mayoría de los lugares más dispositivos, por ejemplo, rampas. Falta arreglar los servicios de transporte, están en mal estado. El ambiente físico no ayuda. No está preparado. En la comunicación no tengo lo que necesito para comunicarme. El ambiente es indiferente y no permite el acceso al mismo." (E12, m, 38a)

Particularmente en el caso de las personas con hipoacusia, se detecta la falta de productos y tecnologías adecuadas para la comunicación, ya sea por la dificultad de acceso al mismo o bien por la presencia de barreras ambientales:

"Para ir al teatro necesito que haya lugares adelantepor el bullicio, es difícil escuchar pero generalmente me acompañan.Al cine voy pero tiene que ser subtitulado. Para ir a lugares voy con alguien, mi novio o mi mamá." (E9, m, 22a)

En general, gran parte de los ambientes, de esparcimiento, laborales e incluso educativos en los que se desenvuelven las personas con discapacidad, presentan dificultades de accesibilidad:

"En los comercios y en la vía pública hay muchas barreras, eso hace que no haya inclusión. El ambiente educacional es inadecuado para la inclusión. La mayoría de los colegios no son accesibles." (E18, m, 22a) 
Los medios de transporte público ofrecen dificultades para la independencia de las personas con discapacidad:

"En los medios de transporte los escalones son muy altos, cuando te bajas no te acercan al cordón, van muy rápido."(E10,m, 35a)

La Ley Nacional No 24.314 "Accesibilidad para personas con movilidad reducida" establece la supresión de barreras físicas urbanas, arquitectónicas y de transporte con la finalidad de lograr la accesibilidad para aqueIlas personas con movilidad reducida. Esta ley detalla el concepto de accesibilidad, de barreras y prevé las condiciones para un medio ambiente accesible. A pesar de su vigencia desde el año 1994, la misma se cumple parcialmente y sumado a la escasa conciencia social al respecto, las personas en situación de discapacidad se enfrentan cotidianamente a obstáculos en la accesibilidad. Esto impacta en su desempeño ocupacional, tal como lo expresó el grupo de estudio.

\section{Los factores contextuales ambientales sociales Servicios, sistemas y políticas.}

Desde hace varios años se encuentran en marcha una serie de políticas, programas y servicios para promover el empleo en personas con discapacidad a nivel nacional, provincial y municipal. En el marco del Ministerio de Trabajo, Empleo y Seguridad Social de la Nación se desarrollan acciones con la finalidad de la inclusión laboral a través de diversos programas para Promover igualdad de oportunidades de empleo, de Inserción Laboral, de Empleo independiente y una Oficina de Empleo. Desde el Ministerio de Trabajo de la Provincia de Buenos Aires funciona el Servicio de Colocación Laboral Selectiva para Personas con Discapacidad.

En general, en el grupo de estudio existe desconocimiento de estos programas y líneas de acción:

"No tengo conocimiento de programas y proyectos para personas con discapacidad; creo que falta mucho por hacer." (E6, m, 50a)

"No conozco, sólo participo en la iglesia." (E10, $\mathrm{m}, 35 \mathrm{a})$
Solamente en un caso, existe cierto conocimiento a partir de experiencias personales:

\begin{abstract}
"Yo en el trabajo en el que estoy, soy funcionario público de la provincia, lo conseguí entrando en un programa de discapacidad que está abocado a incorporar personas con discapacidad en la administración pública, por ese lado sí tenemos ayuda." (E8, h, 40a)
\end{abstract}

En algunas ocasiones sólo se conoce aquellas instituciones en las que se participa, por ejemplo, grupos deportivos o grupos que nuclean a personas con discapacidad con el fin de realizar actividades deportivas o recreativas.

Los entrevistados consideraron que las leyes referidas a garantizar la accesibilidad para personas con movilidad reducida, no se cumplen en su totalidad, existiendo edificios públicos que no son accesibles, o en los que se realizan reformas frente a necesidades puntuales de la persona con discapacidad que habitualmente concurre y hace uso de las instalaciones:

"...creo que a medida que la gente o los que tienen la responsabilidad ven que hay algo que no está bien y se dan cuenta, intentan modificarlo. Así que creo que es una cuestión de prueba y error, de tiempo y de tolerancia." (E8, h, 40a)

En general la creencia es que las leyes existen pero no se cumplen en las diversas áreas, por ejemplo en cuanto a cobertura de obras sociales y sistemas de salud, en el ámbito laboral, en transporte:

"... en transporte de larga distancia no dejan subir más de una persona con discapacidad y si se encuentran dos que quieren viajar en el mismo, uno debe esperar el próximo." (E10, m, 35a)

"No hay cumplimiento de las leyes, en el transporte un poco." (E13, $\mathrm{m}, 2 \mathrm{Oa})$

"Muchas veces evaden las leyes y no se cumplen." (E14, h, 49a) 
"Ahora no hago terapias porque la obra social no me aprobó el año. No tengo ni transporte ni trabajo." (E2O, h, 19a)

Existe un amplio marco legislativo para el tratamiento igualitario de todos los ciudadanos, sin embargo, sigue perpetuándose la exclusión y las desventajas sociales que afrontan cotidianamente las personas con discapacidad.

\section{Actitudes de la familia}

Son varias las perspectivas desde las que se define y comprende a la familia. Actualmente, puede concebirse a la familia como el grupo de personas que interactúan en un mismo espacio, llevando a cabo las funciones biológicas, psicológicas, sociales y culturales básicas. Sus integrantes responden a un compromiso, sea tácito o explícito, de cuidado, protección económica, protección afectiva (Sinche y Suárez, 2006).

La Organización Mundial de la Salud (oms) destaca que no sólo es una unidad básica de organización social, sino que es la más accesible para dar sostén a las acciones preventivas y terapéuticas (Pantano, Núñez y Arenaza, 2012).

La familia se construye a sí misma y da lugar a espacios singulares y a singularidades, a vínculos que exceden a las regulaciones propias de la sociedad y la cultura. Cada familia se crea y recrea generando modalidades vinculares (Rojas citado por Pantano et al., 2012).

Las familias transitan de manera particular los diversos ciclos vitales, los propios del grupo y los individuales de cada integrante; constituyen una red de sostén para la persona con discapacidad. Y así, los entrevistados valoraron el acompañamiento en las diversas instancias vitales:

"El grupo familiar me ayudó siempre, mi mamá, papá y hermana, y es muy importante para mi." (E9, m, 22a)

"Mi familia me ayudó desde pequeño, cuando estaban por fallecer pidió ayuda a mi primo." (E14, h, 49a)
A partir de las entrevistas se deduce que las mayores dificultades de la persona con discapacidad respecto a su familia se plantean en la etapa de la adolescencia y primeros años de la juventud, particularmente, cuando aún los jóvenes no han logrado afianzar un proyecto de vida:

"Si mamá no me lleva a ningún lado, me quedo adentro todo el día. Cuando juega Aldosivi me llevan a la cancha. Me dejan en casa muchas horas." (E18, m, 22a)

"Solo voy a terapias. Estoy todo el día con la computadora y el celular." (E2O, h, 19a)

El apoyo social que ofrece la familia genera bienestar físico y emocional en sus miembros, reduce el estrés que producen algunas situaciones vitales como el caso de la discapacidad y promueve la autoestima, el fortalecimiento de la identidad y, por consiguiente, la construcción subjetiva como sujetos:

"En cuanto a mi familia, amigos y compañeros, todas estas personas han resguardado mi integridad física y emocional (...)"(E10, m, 35a)

\section{Actitudes del entorno educativo}

En el ámbito educativo se generan interrogantes y se plantean diversas experiencias y vivencias en cuanto a las posibilidades de inclusión.

A lo largo del proceso de análisis de las entrevistas quedan explícitas, en las experiencias de gran parte del grupo de estudio, algunas tensiones, que marcaron la vida escolar. Prevalece la concepción de integración escolar, desde el enfoque de las necesidades educativas especiales (NEE), independientemente de la etapa histórica en la que estuvieron escolarizados:

"En cuanto a los docentes, con quienes más dificultad tuve fue con aquellos que se encuentran trabajando en el área de discapacidad, en la escuela especial donde asistía no aceptaban mi integración en escuelas primaria ni en secundaria $(. . .)^{\prime \prime}(\mathrm{Eg}, \mathrm{m}, 22 \mathrm{a})$ 


\section{“(...) vos escuchas muchas veces que los profesores} hablan de vos en el aula, pero después hablan de vos con tu mamá: no mire señora, con $M$ nos hemos esforzado pero no logramos que aprenda." (E11, h, 45a)

El acento estuvo puesto en lo que el alumno no puede, en el déficit, en el rendimiento esperado y no alcanzado. Puede suponerse una representación patologizante de los y las estudiantes. Estas concepciones quedan de manifiesto tanto en experiencias en escuela primaria, secundaria, como en formación superior:

"La persona que está encargada del área de discapacidad en Consejo Escolar le dijo a mi mamá:yo no quiero que mi hija tenga una maestra sorda (...)" (E9, m, 22a)

Sin embargo, las experiencias educativas en el área de los Centros de Formación Profesional y Escuelas de oficios, las actitudes fueron diferentes:

"Muy contenta con el ambiente educativo en el que me encuentro, ya que la profesora es muy agradable y nos ayuda a todos por igual. Me siento en igualdad de condiciones y oportunidades con mis compañeros, aunque sea dentro del aula." $(\mathrm{E} 1, \mathrm{~m}, 43 \mathrm{a})$

"A míla experiencia me gustó, me incluyeron. Me hice amigos" (E4, h, 23a)

Este tipo de experiencias se encuentran centradas en las propias elecciones de cada estudiante, se centran en sus intereses, capacidades y potencialidades, se desarrollan en una etapa más cercana a la adultez. A su vez, el tipo de instituciones en el que se desarrollan estas experiencias se centran en la preparación para el mundo del trabajo y el desarrollo de habilidades específicas, no en cuestiones exclusivas de rendimiento pedagógico.

\section{Actitudes individuales de conocidos, compañeros, colegas, vecinos y miembros de la comunidad.}

La vivencia de las personas del grupo de estudio en relación a las actitudes de personas cercanas, de su entorno inmediato, son coherentes con las experiencias de socialización y participación social. Por ello, se pueden identificar dos grupos de actitudes: positivas, para el caso de personas con discapacidad que participan de actividades en los ámbitos de estudio, laboral, esparcimiento; negativas, en el caso de quienes no participan o en los casos en los que el contexto se vivencia como no inclusivo:

\begin{abstract}
"Migrupo social es el motor que tengo para superar los límites que a veces me pone el contexto, ellos siempre influyen positivamente." (E8, h, 40a)

"La comunidad, vecinos, conocidos no se han dado cuenta de lo que he tenido que pasar." (E1O, $\mathrm{m}, 35 \mathrm{a})$
\end{abstract}

La interacción social genera percepciones positivas de los otros, dando lugar a una retroalimentación en favor de las relaciones interpersonales.

\section{Desempeño ocupacional}

El desempeño de las actividades se manifiesta en tres niveles de análisis: participación ocupacional, desempeño ocupacional y habilidad ocupacional. En el marco de este trabajo se realiza el análisis de los dos primeros niveles.

La participación ocupacional o social se refiere al compromiso personal en situaciones vitales (trabajo y estudio, tiempo libre, autocuidado) y es necesaria para el bienestar. La participación no sólo supone desempeño sino también experiencia subjetiva (Yerxa citado por Kielhofner, 2004). Es tanto personal como contextual; personal refiere a los tipos de participación influidos por los motivos, roles, hábitos, capacidades y limitaciones singulares. Es contextual en la medida que el medio ambiente la facilita o la restringe. 
La discapacidad se manifiesta en el desempeño en las actividades de la vida cotidiana en función de las barreras o condiciones del ambiente. El desarrollo de las actividades varía en cada cultura y sociedad; la discapacidad es una experiencia única para cada persona y, al mismo tiempo, construida cultural, lingüística y socialmente. La discapacidad se construye cuando esa interacción no permite la satisfacción de necesidades y cuando genera limitaciones en el desarrollo de actividades y en la participación.

\section{Áreas principales de la vida}

\section{Trabajo y empleo y Vida económica}

Según datos publicados por el Servicio Nacional de Rehabilitación (SNR), del total de personas mayores de 14 años que cuentan con Certificado Único de Discapacidad (CUD) en la Provincia de Buenos Aires (69.098 personas), solo el $14.92 \%$ trabaja siendo la desocupación en las mujeres del $87.74 \%$ y en los hombres del $82.62 \%$. Los que trabajan lo hacen como empleados u obreros $(66.76 \%)$ y trabajadores por cuenta propia (28.39\%) (Servicio Nacional de Rehabilitación, 2016).

En este contexto, el grupo de estudio destaca las dificultades para encontrar un empleo; solamente cuatro personas se encuentran incluidas laboralmente de manera formal y dos por cuenta propia, una como repostera y otra dictando clases particulares:

\section{“(...) con el trabajo no he tenido posibilidades, aparentemente cuando te entrevistan 'todo bien' y cuando presento la libreta que certifica mi dis- capacidad, nunca me llaman." (E10, m, 35a) \\ "En el ámbito laboral cuesta más (...) como prin- cipal dificultad (pausa) que quizás hay prejuicios respecto al rendimiento laboral de la persona con discapacidad." (E2, m, 21a)}

La posibilidad de autosuficiencia económica depende en gran medida de la pensión por discapacidad que poseen las personas, siendo una expectativa vital tener un trabajo formal y en el caso de los más jóvenes continuar con su formación.
La incorporación al mundo del trabajo permite la plena inclusión, el reconocimiento social y el desarrollo personal, de la confianza y autoestima, transformándose por consiguiente en un tema la salud y bienestar. Es por ello que, la no participación en el mundo laboral tiene consecuencias que exceden ampliamente al problema económico.

\section{Tiempo libre y ocio.}

El tiempo libre se ha estudiado como parte del desempeño ocupacional de la persona, corresponde a una actividad no obligatoria que está intrínsecamente motivada y en la cual se participa durante un tiempo discrecional o libre, es decir, un tiempo no comprometido con ocupaciones obligatorias tales como trabajo, cuidado propio o dormir (American Occupational Therapy Association, 2008). El tiempo libre, considerado como una ocupación, puede abarcar un conjunto de actividades con significados socialmente construidos. Tiene forma, función y significado definidos y observables en un contexto cultural. La forma se refiere a las características visibles de una ocupación, según sean sedentarias, solitarias, sociales, intelectuales, físicas, etc. La función corresponde al objetivo que se persigue, sea esparcimiento o recreación y ocio. El significado corresponde a la vivencia interna que resulta de participar en determinadas ocupaciones; es posible relacionar la selección y participación en actividades de tiempo libre con las expectativas de la persona y/o su entorno, las experiencias previas, las creencias y valores, etc. (Muñoz y Salgado, 2006)

El grupo de estudio realiza actividades de tiempo libre, especialmente prácticas deportivas y sociales, en general, en el marco de propuestas de ONG o grupos para personas con discapacidad. Estas actividades son elegidas libremente según intereses personales, resultan confortables y cumplen una función recreativa o de esparcimiento, para satisfacer necesidades de interacción social. Algunos testimonios dan cuenta de ello:

"A Punto Encuentro voy los sábados. Con los chicos del trabajo no salgo; sí con los de la escuela. Algunas veces juego al fútbol en el barrio, con conocidos de la familia." (E4, h, 23a)

"Participo en un grupo de trote, corro maratón y me gustaría trabajar." (E9, m, 22a) 
"Voy a la pileta, voy a recitales, actividades en la iglesia, playa, plaza y me gustaría trabajar."(E10, $\mathrm{m}, 35 \mathrm{a})$

La necesidad de cultivar amistades y mantener relaciones sociales es percibida como un aspecto beneficioso y que resulta fundamental sostener en el tiempo:

"Me gustaría participar con gente del mismo ambiente o discapacidad para poder charlar, hablar de mi vida, cómo la fui llevando y la fui peleando hasta ahora." (E15, h, 50a)

En algunos casos se expresa el interés de participar en actividades de tipo cultural:

\section{“(...) me gustaría participar en alguna actividad} cultural relacionada con el teatro o la música, eso me gustaría." (E8, h, 40a)

\section{Inclusión desde la perspectiva de los participantes}

Inclusión es potenciar al máximo la participación de las personas en las diferentes esferas de la vida.

Las deficiencias solamente adquieren significado cuando se convierten en experiencias por la interacción social. No todo cuerpo con deficiencia, vivencia la discriminación, la opresión o la desigualdad por la discapacidad, pudiendo existir una relación de dependencia entre el cuerpo con deficiencias y el grado de accesibilidad de una sociedad. Cuanto mayores sean las barreras sociales, mayores serán las restricciones de participación impuestas a las personas con discapacidad (Diniz, Barbosa y Santos, 2009).

La exclusión supone, para Burchardt (citado por Barton, 2009), un complejo proceso de negación, de falta oportunidades y relaciones sociales; de irrupción a la identidad y dignidad de la persona; marginación respecto al ejercicio de la ciudadanía, los derechos civiles y el respeto humano. Su impacto en la vida de la persona puede ser acumulativo a lo largo de las diversas etapas de la vida; sus consecuencias son profundas no sólo en la vida de la persona excluida sino en la sociedad.
La concepción de inclusión que sostienen los participantes se asocia a la capacidad para realizar actividades, a la autonomía, a la productividad, en el sentido de poder desempeñarse con independencia y en igualdad de condiciones.

"Cuando puede hacer todo como todas las personas. Hay que enseñarle a la gente de inclusión." (E19, h, 20a)

"Una persona está incluida cuando puede hacer de todo y no necesita ayuda." (E2O, h, 19a)

"Una persona está incluida cuando puede hacer su vida normalmente, no tiene impedimentos. Es decir, si le dan ganas de ir a un lugar puede hacerlo si quiere, no porque tenga una discapacidad deja de hacer vida normal." (E2, m, 21a)

En algunos de los testimonios se aprecia que la inclusión se da como exigencia adaptativa de la persona, no como resultado de la interacción sociedad-sujeto. Ante un contexto desfavorable es la persona con discapacidad la que tiene que adaptarse:

"El cursar te despeja un poco la mente también. Te ayuda a adaptarte a la sociedad. Vos haces el curso y el laburo pero siempre hay una responsabilidad de por medio y hay que adaptarse a la sociedad, eso lo comprendo."( $\left.\mathrm{E}_{5}, \mathrm{~h}, 21 \mathrm{a}\right)$

Y las actitudes sociales se revelan en algunas expresiones:

"La sociedad piensa respecto de la discapacidad como algo malo, como un defecto no como una virtud. La gran mayoría de las personas discriminan, no aceptan la discapacidad. Algunas otras síla aceptan." (E6, m, 50a)

"La gente mayor, en general, te tiene más lastima o te mira con mucho respeto."(E8, h, 40a)

"La mayoría muestra indiferencia, no se acercan, algunas otras sí se acercan. Piensan en la falta, en la lástima." (E9, m, 22a) 


\begin{abstract}
"Las personas creen que es algo lejano que nunca les va a ocurrir, que ellas están al margen de la discapacidad, piensan que somos pobrecitos. El ambiente no te incluye te deja de lado. La mayoría es indiferente, las personas miran por su propio bienestar." (E10, m, 35a)
\end{abstract}

"Algunos se acercan y otros son indiferentes; son más los indiferentes." (E13, m, 22a)

Las actitudes sociales hacia las personas con discapacidad pueden resultar obstáculos más importantes para su inclusión en la comunidad que los derivados de la propia deficiencia o condición de discapacidad.

La percepción acerca de la inclusión de las/los entrevistados queda reducida al esfuerzo personal, en la esfera privada, asociada a la capacidad de independencia en el desempeño cotidiano, sin el sostén social necesario para una participación plena en igualdad de condiciones y oportunidades.

La participación es un concepto dinámico, que se dirige a la construcción de sujetos y sociedades por cuanto es una fuerza o poder que instituye relaciones en todas las esferas del ser humano. Si existe la capacidad de promover sentido de lo público (ciudadanía) en el que subyacen valores como la equidad, la solidaridad, el respeto y la tolerancia, entonces se crean las condiciones favorables para la inclusión social (Prieto, 2004).

\section{Discusión}

Desde la perspectiva de las personas en situación de discapacidad, los factores contextuales que se relacionan con la inclusión social y el desempeño ocupacional son:

De los factores personales, aquellos inherentes a las propias actitudes en las diversas esferas de la vida cotidiana, destacándose el sentido de compromiso, la responsabilidad y el estado de ánimo positivo. Estos factores coinciden con otros hallazgos (Vidaña, 2017) que destacan actitudes personales como la confianza en las propias capacidades para afrontar situaciones desafiantes y la búsqueda de apoyo social como facilitadores de la participación ocupacional.
Entre los factores contextuales ambientales físicos se destacan las dificultades en el transporte público y en la accesibilidad física y comunicacional a los espacios abiertos y cerrados, donde se realizan las actividades de esparcimiento, laborales y/o educativas. En consonancia a estos hallazgos, un estudio (Hurtado, Aguilar, Antó, Sandoval, Peña, Díaz, 2012) considera que las múltiples barreras que afectan la inclusión social sugieren que se debe abordar esta problemática desde la estructura y organización social del entorno en que se desempeña la persona y no solamente desde su deficiencia. Las barreras edilicias y en los medios de transporte públicos,si bien reducen la autonomía en la movilidad, la falta de accesibilidad al entorno físico y a la información y comunicación fue el factor restrictivo para la participación en la vida comunitaria, social y cívica y en la formación profesional de las personas con discapacidad motriz de dicho estudio.

Los factores contextuales ambientales sociales constituyen el foco del análisis dado que los participantes los identificaron espontáneamente a largo de sus relatos. Entre los factores favorecedores de inclusión se destacan las actitudes de los familiares y de los docentes de Centros de Formación Profesional. Pallisera (2011) refuerza con su estudio la idea de fortalecer la función de las escuelas secundarias para facilitar la inclusión social y laboral de los jóvenes en situación de discapacidad. Asimismo, plantea como aspecto poco estudiado el papel de la familia en el proceso de transición de la escuela a la vida adulta activa y destaca que la implicación familiar, el apoyo y las expectativas adecuadas inciden positivamente en la inclusión laboral de los hijos con discapacidad.

Entre los factores que obstaculizan la inclusión se reconocieron el desconocimiento de algunas políticas inclusivas disponibles; pero especialmente aparece la necesidad y el reclamo frente a la falta o insuficiencia de servicios, sistemas y políticas vinculados al empleo y a la cobertura de salud. Resultados similares se presentan en el trabajo de revisión (Serrano, Ramírez, Miranda, Camargo, Guerra, Clavijo, 2013) que identifica a los principales factores limitantes de la participación a aquellos de orden físico, político, personal y social, que limitan la inclusión de las personas con discapacidad física en escenarios relacionados con las actividades laborales y con el acceso a servicios de salud y de educación. Se identifican la ausencia, escasa efectividad o desconocimiento de políticas que promuevan la participación 
laboral y la actitud negativa frente a la discapacidad, falta de apoyo o desconocimiento acerca del manejo de la discapacidad.

Este último aspecto social es otro de los factores actitudinales que obstaculiza la plena inclusión desde las perspectivas de las personas con discapacidad entrevistadas, como las actitudes negativas de docentes y profesionales en el ámbito educativo (escuelas primarias, secundarias y terciarias). Las actitudes de los profesionales condicionan con frecuencia las oportunidades reales de inclusión, por ello, la necesidad de implementar acciones de formación y de sensibilización permanente dirigidas a los docentes (Pallisera, 2011).

Estos factores contextuales se vinculan entre sí configurando los escenarios particulares en cada esfera de la vida. De las experiencias subjetivas relatadas emerge el sentido de inclusión como la capacidad de sentirse productivo y autónomo, en igualdad de condiciones. En cuanto al trabajo, constituye el área de mayor preocupación de las personas con discapacidad debido al desempleo y a la precariedad de los empleos a los que acceden. Las personas jóvenes o adultas esperan tener una vida productiva y las actividades de tiempo libre u ocio y deporte ocupan el espacio vacío que dejan las actividades laborales deseadas. En el estudio de Arévalo y Yáñez (2016) se describe que persiste la participación segregada en actividades comunitarias de ocio y tiempo libre donde sólo se relacionan entre sí personas vinculadas a la discapacidad. Los autores sostienen que para lograr una participación en actividades más inclusivas, es necesario que los organismos implicados refuercen los conceptos de igualdad de oportunidades e inclusión social en el ámbito del ocio y no sólo se enfaticen dichos conceptos en el ámbito de la educación y el trabajo, el tiempo libre y ocio es un área que promueve la salud y el desarrollo personal.

Respecto de las barreras sociales, al ignorar los cuerpos con deficiencias, provocan la experiencia de la desigualdad. La deficiencia no es un atributo del cuerpo, sino resultado de sociedades no inclusivas. Diniz et al. (2009) considera que las deficiencias solamente adquieren significado cuando se convierten en experiencias por la interacción social. No toda persona en situación de discapacidad vivencia la discriminación, la opresión o la desigualdad por la discapacidad misma, ya que hay una relación de dependencia entre el cuerpo con deficiencias y el grado de accesibilidad de una sociedad. Cuanto mayores sean las barreras sociales, mayores serán las restricciones de participación impuestas a los individuos con deficiencias.

Por tanto, quedan abiertas varias líneas de investigación, vinculadas a cada uno de los factores estudiados, al significado atribuido a las actividades de tiempo libre y ocio y a la concepción de inclusión. No obstante, en este estudio un aspecto recurrente estuvo constituido por las dificultades en la inclusión laboral.

Desde las vivencias experimentadas por el grupo de estudio se interpreta que persisten resabios del modelo médico, éstas se caracterizan por la conjugación de varios conceptos vinculados a la etiología, patología, síntomas y manifestaciones corporales, generando una comprensión lineal de la discapacidad.

En un intento de análisis se puede suponer que el concepto de discapacidad y el propio modelo médico han contribuido a la formación de representaciones sociales acerca de la discapacidad que conllevan a pensar a la persona como portadora de desventajas y limitaciones que son consecuencia de una patología física y/o psíquica persistente en el tiempo y que provocan dependencia. La mirada se dirige a la patología, a las limitaciones, al funcionamiento de determinados órganos, por consiguiente se pierde de vista la integralidad de la persona, se sacrifica el todo por las partes, dando un lugar de relevancia a la condición de dependencia permanente.

La consecuencia de esta concepción consiste en suponer que las personas con alguna discapacidad no se encuentran en condiciones de generar sus propios proyectos vitales, provocando una cadena de situaciones de dependencia; es en esta sociedad en la se constituyen como sujetos y desarrollan su personalidad.

El conjunto de la terminología adoptada por este modelo ha permitido en algún sentido unificar criterios en el campo de la medicina, rehabilitación, política. Como consecuencia de estas clasificaciones terminológicas y su popularización, se ha generado, socialmente, una representación acerca de cómo la gente comprende, califica $y$, por ende, estigmatiza a las personas con discapacidad. Creando significados sobre los déficit y sobre los defectos de los demás. El modelo médico lleva implícitas prácticas de significación y efectos perjudiciales en los proyectos vitales de las personas con 
discapacidad, en el seno de sus familias y en el entorno social. Significaciones que aún hoy a pesar del cambio del paradigma médico al social de la discapacidad, persisten y contaminan el imaginario social.

\section{AgRADECIMIENTO}

Este artículo es producto de dos años de trabajo en equipo en el contexto de la Universidad Nacional de Mar del Plata; y no hubiera podido concretarse sin la colaboración desinteresada de las personas que accedieron a participar de las entrevistas.

\section{REFERENCIAS}

American Occupational Therapy Association (AOTA) (2008) Occupational Therapy Practice Framework: Domain and Process

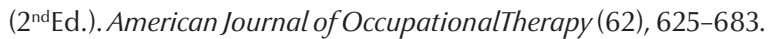
[Traducción] Ávila Álvarez, A., Martínez Piédrola, R., Matilla Mora, R., Máximo Bocanegra, M., Méndez Méndez, B., Talavera Valverde, M. A.,Viana Molde, I. (trad.) (2010). Marco de trabajo para la práctica de la Terapia Ocupacional: Dominio y proceso. ( $2^{\text {a }}$ ed.). Recuperado de http://www.terapia-ocupacional.com/ aota2010esp.pdf

Arévalo, M. y Yáñez, J. (2016). Factores contextuales que influyen en la participación comunitaria en actividades de ocio y tiempo libre en personas en situación de discapacidad física. Revista Chilena de Terapia Ocupacional, 16(2), 163-173. doi:10.5354/07195346.2016.44760

Barton, L. (2009). La posición de las personas con discapacidad. ¿Qué celebrar y por qué celebrarlo? ¿Cuáles son las consecuencias para los participantes? En P. Brogna (Ed.). Visiones y revisiones de la discapacidad (pp. 123-136). México: Fondo de Cultura Económica.

Coffey, A. (2003) Encontrar el sentido a los datos cualitativos. Estrategias complementarias de investigación. México: Contus.

Diniz, D., Barbosa, L. y dos Santos, W. R. (2009). Discapacidad, Derechos Humanos y Justicia. SUR. Revista Internacional de Direitos Humanos, 6(11), 65-77. Recuperado de https://dx.doi.org/10.1590/ S1806-64452009000200004

Hurtado Floyd, M., Aguilar Zambrano, J., Mora Antó, A., Sandoval Jiménez, C., Peña Solórzano, C., León Díaz, A. (2012). Identificación de las barreras del entorno que afectan la inclusión social de las personas con discapacidad motriz de miembros inferiores. Revista Salud Uninorte, 28(2), 227-237. Recuperado de http://rcientificas.uninorte.edu.co/index.php/salud/article/ viewArticle/913

Kielhofner, G. (2004). Modelo de Ocupación Humana. Teoría y Aplicación. $3^{\mathrm{a}}$ ed. Buenos Aires, Argentina: Editorial Médica Panamericana.
Ley $N^{\circ} 24.314$ Accesibilidad de personas con movilidad reducida. Modificación de la Ley N²2.431. Sancionada: Marzo 15 de 1994. Senado y Cámara de Diputados de la Nación Argentina.

Ley No 26.378 Ratificación de la Convención sobre los Derechos de las Personas con Discapacidad (A/RES/61/106 Naciones Unidas). Sancionada: Mayo 21 de 2008. Promulgada: Junio 6 de 2008. Senado y Cámara de Diputados de la Nación Argentina.

Muñoz Espinosa, I., Salgado Gómez,P. (2006). Ocupaciones de tiempo libre: una aproximación desde la perspectiva de los ciclos vitales, desarrollo y necesidades humanas. Revista Chilena de Terapia Ocupacional, (6), 39-45. doi:10.5354/0719-5346.2010.110

Organización Mundial de la Salud. (2001). Clasificación Internacional del Funcionamiento, de la Discapacidad y de la Salud (CIF).Madrid: IMSERSO. Recuperado de http://www.imserso.es/InterPresent2/ groups/imserso/documents/binario/435cif.pdf

Organización de Naciones Unidas. (2006). Convención Internacional sobre los Derechos de las Personas con Discapacidad. A/RES/61/106. New York: Asamblea General ONU. Recuperado de http://www. un.org/disabilities/documents/convention/convoptprot-s.pdf

Pantano, L., Núñez, B. y Arenaza, A. (2012). ¿Quénecesitan las familias de las personas con discapacidad?: investigación, reflexiones y propuestas. Buenos Aires: Lugar Editorial.

Palacios, A. (2008). El modelo social de discapacidad: orígenes, caracterización y plasmación en la Convención Internacional sobre los Derechos de las Personas con Discapacidad. Madrid: Cinca. Recuperado de http://www.cermi.es/es-ES/ColeccionesCermi/

Pallisera Díaz, M. (2011). La inclusión laboral y social de los jóvenes con discapacidad intelectual. El papel de la escuela. Revista Interuniversitaria de Formación de Profesorado, 25(1), 185-200. Recuperado de http://www.redalyc.org/src/inicio/ArtPdfRed. jsp?iCve $=27419147011$

Prieto Rodríguez, A. (2004). La limitación en la participación como discapacidad política. En C. Cuervo Echeverri, A. Trujillo Rojas, D. R. Vargas Pineda, B. Mena Bejarano y L. Pérez Acevedo (Ed.). Discapacidad e Inclusión Social (pp. 121-136). Bogotá: Universidad Nacional de Colombia.

Serrano Ruíz, C. P., Ramírez Ramírez, C., Abril Miranda, J.P., Ramón Camargo, L. V., Guerra Urquijo, L. Y., Clavijo González, N. (2013). Barreras contextuales para la participación de las personas con discapacidad física. Revista de la Universidad Industrial de Santander. Salud, 45(1), 41-51. Recuperado de http://www.redalyc. org/articulo.oa?id=343833959006

Servicio Nacional de Rehabilitación. Anuario Estadístico sobre discapacidad 2016. Buenos Aires: SNR. Recuperado de https://www.snr.gob. ar/wp-content/uploads/2017/08/Anuario-2016-FINALRE.pdf

Sinche, E. E. y Suárez Bustamante, M. (2006). Introducción al estudio de la dinámica familiar. RAMPA, 7(1), 38-47. Recuperado de http:// www.idefiperu.org/RAMNRO1/RAMPA\%20V1N1\%20Parte2.pdf

Valles, M. S. (1999). Técnicas cualitativas de Investigación social. Madrid: Síntesis. 
Vidaña Moya, L (2017). La participación ocupacional como vía de inclusión social en personas con discapacidad física: analizando la relación entre ocupación, entorno y bienestar. Tesis doctoral. Universidad Autónoma de Barcelona, Cataluña, España. Recuperado de https://ddd.uab.cat/record/188088?ln=es 